http://dx.doi.org/ 10.22319/rmcp.v9i1.4324

Artículo

\title{
Endometritis subclínica y tasa de gestación en vacas lecheras en México
} Subclinical endometritis and pregnancy rate in dairy cows in Mexico

José Luis Barajas Merchan ${ }^{\mathrm{a}}$,

Joel Hernández Cerón ${ }^{\mathrm{a}^{*} \text {, }}$

Andrés García Alfonso ${ }^{a}$,

Enrique Martínez Bárcenas ${ }^{\mathrm{a}}$,

Noé Orlando Juárez Lópeza

Mario Adán Bedolla Alva ${ }^{\text {, }}$

Rodolfo Luzbel de la Sota ${ }^{c}$

a Departamento de Reproducción, Facultad de Medicina Veterinaria y Zootecnia, Universidad Nacional Autónoma de México. Ciudad de México, México.

b Departamento de Patología, Facultad de Medicina Veterinaria y Zootecnia, Universidad Nacional Autónoma de México, Ciudad de México, México.

${ }^{\mathrm{c}}$ Facultad de Ciencias Veterinarias, Universidad Nacional de la Plata, La Plata, Argentina.

*Autor de correspondencia: jhc@unam.mx

\section{Resumen:}

En el presente estudio se determinó la prevalencia de endometritis subclínica y su efecto en la tasa de gestación en vacas lecheras en manejo intensivo. Entre los días 35 y 45 posparto se seleccionaron 237 vacas sin patologías uterinas (vacas con útero involucionado y sin secreciones vaginales anormales); a todas ellas se les tomó una muestra citológica de endometrio mediante la técnica de Cytobrush. Los frotis se procesaron con la tinción de Diff- 
Quick. Se contaron un total de 200 células y se determinó la proporción de células polimorfo nucleares (PMN). Las vacas con $\geq 6 \%$ de PMN se consideraron con endometritis subclínica. Cuarenta y tres por ciento (103/237) de las vacas presentó endometritis subclínica. Las vacas con puerperio patológico tuvieron mayor probabilidad de padecer endometritis subclínica que las vacas con puerperio normal (60 vs $36 \%$, respectivamente; razón de probabilidad= 2.69). Las vacas normales tuvieron mayor tasa de gestación en el primer servicio que la vacas con endometritis subclínica (31.4 vs $20.0 \%$; respectivamente; razón de probabilidad=2.02) y la proporción de vacas gestantes en el día 120 posparto fue mayor en las vacas normales que en las vacas con endometritis subclínica (51.6 vs $37.9 \%$, respectivamente; razón de probabilidad $=1.98)$. Se concluye que la prevalencia de endometritis subclínica en vacas lecheras entre los días 35 y 45 posparto es de $43 \%$, lo cual disminuyó en un $11.4 \%$ la tasa de gestación en el primer servicio y en un $13.7 \%$ la tasa de gestación acumulada en el día 120 posparto.

- Palabras clave: Endometritis subclínica, Ganado lechero, Fertilidad.

\section{Abstract:}

The present study determined the prevalence of subclinical endometritis and its effect on pregnancy rate in intensively managed dairy cows. Between d 35 and 45 postpartum, 237 cows with no uterine pathologies judged to have completed uterine involution and no abnormal vaginal secretions were selected. Endometrial cytology samples were taken from each cow using the Cytobrush technique, and smears were stained with Diff-Quick. A total of 200 sample cells were counted and the proportion of polymorphonuclear leukocytes (PMN) was estimated. Cows with $\geq 6 \%$ PMN were considered to have subclinical endometritis; $43 \%$ (103/237) of cows sampled met this criterion. Cows with pathological puerperium had a higher probability of undergoing subclinical endometritis than cows with normal puerperium (60 vs $36 \%$, respectively; odds ratio= 2.69). Cows not presenting subclinical endometritis had a higher pregnancy rate at first service than cows with this condition (31.4 vs $20.0 \%$, respectively; odds ratio= 2.02), and the percentage of pregnant cows at d 120 postpartum was higher in cows not experiencing subclinical endometritis than in cows with this condition (51.6 vs $37.9 \%$, respectively; odds ratio=1.98). It concludes that $43 \%$ of cows suffered subclinical endometritis between d 35 and 45 postpartum, which decreased pregnancy rate at first service, as well as cumulative pregnancy rate at d 120 postpartum.

- Key words: Subclinical endometritis, Dairy cows, Pregnancy rate.

Recibido el 13/11/2016. 
Aceptado el 2/04/2017.

\section{"Introducción N}

En el ganado lechero las patologías uterinas posparto alargan el periodo del parto al primer servicio, disminuyen la tasa de gestación en el primer servicio, alargan el periodo del parto a la primera ovulación, están asociadas con pérdidas embrionarias y aumentan el porcentaje de vacas eliminadas del hato ${ }^{(1-3)}$. Dentro de las patologías uterinas posparto están la retención de las membranas fetales, metritis puerperal, metritis, endometritis y endometritis subclínica. Esta última afecta entre 20 y $40 \%$ de las vacas, reduce la tasa de gestación en el primer servicio y aumenta el porcentaje de pérdidas embrionarias y fetales ${ }^{(4-6)}$.

La endometritis subclínica se define como la inflamación del endometrio sin signos externos de enfermedad ${ }^{(4)}$. Así, al ser una condición que pasa desapercibida, las vacas afectadas terminan su periodo voluntario de espera y se integran a los programas de inseminación. Debido a la dificultad para establecer un diagnóstico de la endometritis subclínica con base en algún indicador externo, el diagnóstico se fundamenta en la presencia de polimorfo nucleares (PMN) en muestras de células endometriales obtenidas mediante lavados uterinos o por medio de la recolección de células con un cepillo endocervical de uso humano (técnica de Cytobrush) $)^{(6-8)}$.

La variación de la prevalencia de endometritis subclínica está asociada con las características de los hatos, con los días posparto en que se hace el diagnóstico y con el punto de corte de la proporción de PMN de las muestras. La prevalencia en todos los estudios fluctúa entre $20 \mathrm{y}$ $40 \%$ e influye negativamente en la tasa de gestación, particularmente en el primer servicio $^{(7,9,10)}$. En México se desconoce la prevalencia de endometritis subclínica, su efecto en la tasa de gestación y sus factores de riesgo. Dada la similitud en los sistemas de producción intensiva de leche en bovinos a nivel global, se podría asumir que en México la prevalencia de endometritis subclínica y su efecto en la fertilidad serían similares a lo publicado en otros países, lo cual es probable; sin embargo, la prevalencia de enfermedades reproductivas es diferente entre países y regiones, por tanto es deseable generar referencias nacionales que permitan tener datos objetivos de los problemas que enfrentan los productores de leche, y que sirvan para dirigir la investigación científica que se hace en las universidades en instituciones especializadas. Por tanto, el objetivo del presente estudio fue determinar la prevalencia de endometritis subclínica en vacas lecheras en manejo intensivo y su efecto en 
la tasa de gestación. Se hipotetizó que la endometritis subclínica afecta a una proporción significativa de las vacas lecheras de hatos de la región central de México y provoca menor tasa de gestación en el primer servicio.

\section{Material y métodos}

\section{- Manejo de los hatos •}

El estudio se realizó en el Complejo Lechero de Tizayuca, Hgo., localizado en el altiplano central de México. El clima de la región es templado subhúmedo, con una temperatura anual promedio de $15.5^{\circ} \mathrm{C}$ y una precipitación pluvial media anual de $861 \mathrm{~mm}$. Se utilizaron vacas de 16 establos de manejo similar. Los animales estuvieron en estabulación en corrales con cubículos de libre acceso, la alimentación consistió en una ración totalmente mezclada, balanceada de acuerdo con las recomendaciones del $\mathrm{NRC}^{(11)}$. La producción de leche promedio por vaca fue entre 8,000 y $9,000 \mathrm{~kg}$ por lactación.

La revisión reproductiva para el diagnóstico y tratamiento de las patologías del puerperio se realizó en los primeros 10 días posparto, y se aplicaron los tratamientos farmacológicos específicos para cada caso. Las vacas se diagnosticaron sanas (vacas limpias) cuando por vía transrectal se apreciaba que el útero estaba involucionado y no se observaron secreciones vaginales anormales. El periodo voluntario de espera fue de 50 días. Las vacas se identificaron en estro mediante la observación de la aceptación de la monta y se inseminaron por un mismo técnico bajo el programa am-pm/pm-am. El semen fue de toros de fertilidad probada, seleccionados de acuerdo con los programas de mejoramiento genético de cada hato.

\section{- Diseño del estudio •}


El manejo de los animales durante el experimento fue aprobado por el Consejo Académico del Posgrado en Ciencias de la Producción y de la Salud Animal de la Universidad Nacional Autónoma de México.

Entre los días 35 y 45 posparto se seleccionaron 237 vacas de diferente número de parto, las cuales estaban clínicamente sanas, su útero estaba totalmente involucionado y no mostraron secreciones vaginales anormales visibles después de palparlas por vía transrectal. Se obtuvieron muestras para citologías uterinas mediante la técnica de Cytobrush; el Cytobrush es un cepillo endocervical de uso humano, el cual se introdujo hasta la curvatura mayor del cuerno uterino mediante la técnica descrita por Plöntzke et $a l^{(8)}$ y modificada por Madoz et $a l^{(6)}$. Después de retirarlo, se hizo un frotis en un portaobjetos, el cual se fijó en inmersión en alcohol durante 60 min. Las laminillas se tiñeron mediante la técnica Diff Quick. Se contaron, mediante microscopía 200 células endometriales y se obtuvo la proporción de PMN.

El punto de corte del porcentaje de PMN que correspondió con una disminución de la eficiencia reproductiva fue obtenido mediante el análisis de la curva $\mathrm{ROC}^{(12)}$, para lo cual se utilizó el paquete estadístico y de gráficos SigmaPlot $10^{(13)}$. La curva ROC (relative operating characteristics) es la representación de la razón de verdaderos positivos (VPR) frente a la razón de falsos positivos (FPR), según se varía el umbral de discriminación (valor a partir del cual decidimos que un caso es un positivo). Se graficó la sensibilidad y la especificidad para cada porcentaje de PMN posible en una curva ROC con la mediana de 128 días de IPC (días de vaca vacía) en un grupo de 100 vacas, y se determinó como umbral para clasificar a una vaca con endometritis subclínica cuando se encontraron $\geq 6 \%$ de PMN (área bajo la curva $=0.56$; error estándar $=0.06$; IC del $95 \%=0.44-0.68 ; P=0.03)^{(6,7)}$.

\section{- Análisis estadístico •}

El efecto de la endometritis subclínica en la tasa de gestación al primer servicio y en la tasa acumulada de gestaciones en el día 120 posparto, así como los factores de riesgo como el número de partos (primíparas y multíparas) y el tipo de puerperio [normal o patológico (vacas que padecieron cualquiera de las siguientes condiciones: retención placentaria, metritis o secreción purulenta por vagina)] se analizaron mediante regresión logística. Los días del parto al primer servicio se compararon por análisis de $\operatorname{varianza}^{(14)}$. 


\section{Resultados}

La curva ROC identificó como punto de corte a $\geq 6 \%$ PMN (Figura 1). Utilizando el punto de corte obtenido con la curva ROC, la prevalencia de endometritis subclínica fue de $43 \%$ (103/237). Los días del parto al primer servicio fueron similares $(P>0.05)$ entre las vacas con endometritis subclínica $(65.7 \pm 1.9)$ y en las vacas normales $(63.9 \pm 1.6)$. Las vacas con endometritis subclínica tuvieron menor tasa de gestación al primer servicio y menor tasa de gestación acumulada en el día 120 posparto en comparación con las vacas normales (Cuadro 1). Las vacas con antecedentes de puerperio patológico tuvieron mayor probabilidad de padecer endometritis subclínica $(60$ vs $36 \%$; puerperio patológico y normal, respectivamente; razón de probabilidad $2.69 ; P<0.05)$. El número de partos y el hato no influyeron en la prevalencia de endometritis subclínica $(P>0.10)$.

Figura 1. Curva ROC para estimar el porcentaje de corte de PMN (6 \%) para las vacas estudiadas

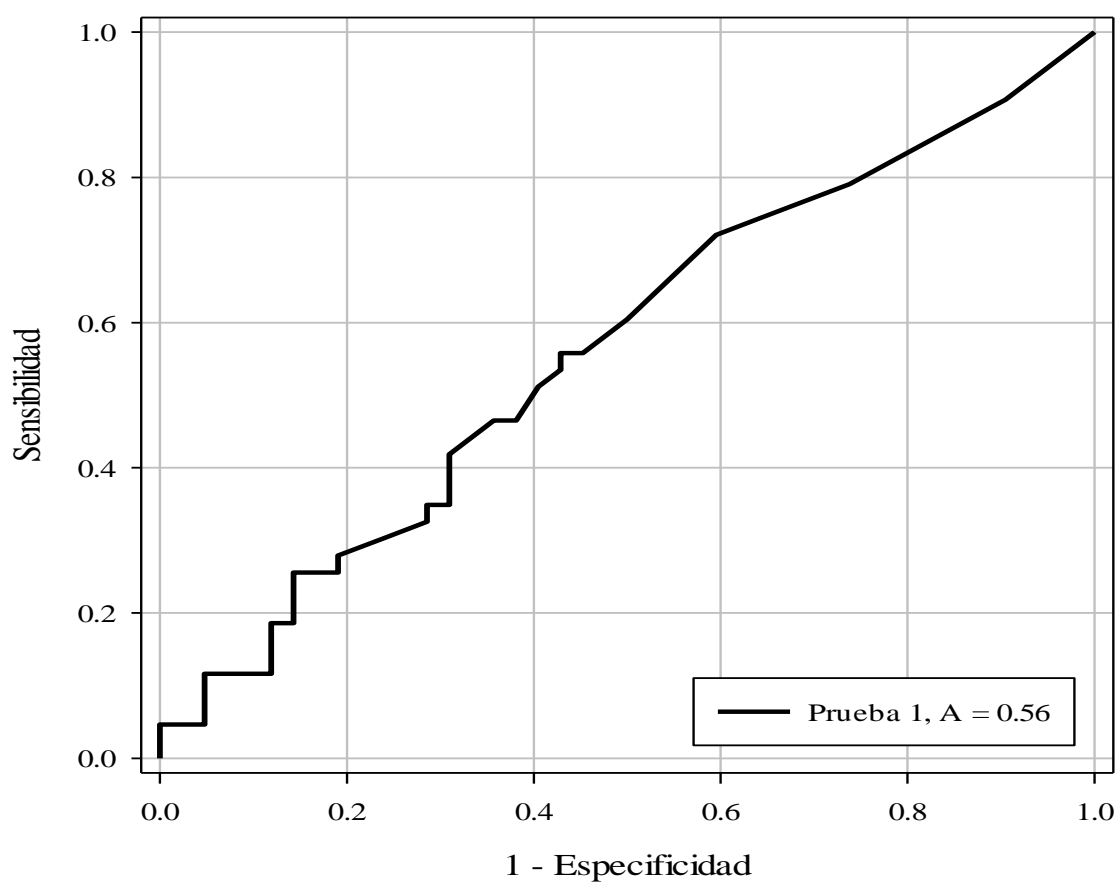


Cuadro 1. Razón de probabilidad de gestación en el primer servicio y de gestaciones acumuladas al día 120 posparto en vacas con y sin endometritis subclínica

\begin{tabular}{lcccccc}
\hline $\begin{array}{l}\text { Tasa de } \\
\text { gestación }\end{array}$ & $\begin{array}{c}\text { Endometritis } \\
\text { subclínica }\end{array}$ & $\mathbf{n}$ & $\begin{array}{c}\text { Tasa de } \\
\text { gestación }\end{array}$ & $\begin{array}{c}\text { Razón de } \\
\text { probabilidad }\end{array}$ & IC 95\% & $\boldsymbol{P}$ \\
\hline $\begin{array}{l}\text { Primer } \\
\text { servicio }\end{array}$ & No & 124 & 31.4 & 2.02 & $1.04-3.92$ & 0.03 \\
& $\mathrm{Si}$ & 95 & 20.0 & Referencia & & \\
$\begin{array}{l}\text { Día } 120 \\
\text { posparto }\end{array}$ & No & 124 & 51.6 & 1.98 & $1.11-3.53$ & 0.02 \\
& $\mathrm{Si}$ & 95 & 37.9 & Referencia & & \\
\hline
\end{tabular}

\section{| Discusión}

Este trabajo representa el primer estudio sobre la prevalencia de endometritis subclínica en ganado lechero en México. En concordancia con la hipótesis de trabajo planteada, $43 \%$ de las vacas diagnosticadas sanas después de su manejo clínico posparto, tuvieron endometritis subclínica, con una disminución en la eficiencia reproductiva. Dicho parámetro está dentro de los rangos publicados en otros estudios ${ }^{(5-7,15)}$. Un dato interesante del presente trabajo es que el tipo de animales muestreados fueron todas las vacas independientemente de su tipo de puerperio; así, se incluyeron vacas que padecieron retención placentaria, metritis y endometritis, mismas que se agruparon en el análisis como vacas con puerperio patológico. Es relevante, también, el tiempo posparto en que se realizó el diagnóstico; en el presente estudio se realizó entre los días 35 y 45 posparto, tiempo en que regularmente ya se completó la involución uterina y la vaca ya eliminó los procesos infecciosos, ya sea basada en sus propios mecanismos de defensa o favorecida por el tratamiento antimicrobiano. Cabe señalar que la prevalencia de endometritis subclínica tiende a ser menor entre más lejos esté la vaca del parto ${ }^{(16)}$, por tanto este dato indica que en los hatos estudiados, el problema de la endometritis subclínica tiene una prevalencia alta, ya que aproximadamente la mitad de las vacas $(43 \%)$ tuvo un proceso inflamatorio en el endometrio muy cerca del final del periodo voluntario de espera (50 días).

En el presente estudio no se determinaron los agentes infecciosos involucrados en el proceso; sin embargo, en otros trabajos el aislamiento de los agentes infecciosos involucrados ha arrojado resultados variables y no siempre se han aislado bacterias ${ }^{(6)}$, pero la bacteria aislada 
con mayor frecuencia es Trueperella pyogenes ${ }^{(17,18)}$.

Se observó mayor probabilidad de padecer endometritis subclínica en las vacas con antecedentes de puerperio patológico; esta observación es congruente, ya que los procesos inflamatorios en el endometrio dejan secuelas; además, cuando hay daño en el endometrio hay mayor producción de citoquinas $\mathrm{y}$, en consecuencia, mayor migración de $\mathrm{PMN}^{(19)}$. Viendo a la endometritis subclínica desde una perspectiva práctica, la estrategia para disminuir su prevalencia consiste en disminuir la incidencia de patologías del puerperio y, aunque la etiología de estas patologías es multifactorial, una constante que determina en gran medida la evolución de las vacas durante el puerperio son los cambios metabólicos ocasionados por la pérdida de condición corporal posparto. Las vacas más susceptibles a las patologías uterinas posparto son las que pierden mayor condición corporal después del $\operatorname{parto}^{(9,20,21)}$.

Cabe señalar que la endometritis subclínica no es una patología exclusiva del ganado lechero, sino también se ha diagnosticado en bovinos en sistemas de producción de carne ${ }^{(22,23)}$. Ricci et $a l^{(23)}$ encontraron en vacas Piamontesas $31 \%$ de prevalencia y un efecto negativo en el intervalo parto a la concepción. En ganado encastado Cebú-Holstein en pastoreo también se observan prevalencias de 26 a $30 \%$ y afectación de la fertilidad ${ }^{(24,25)}$.

En el presente estudio tanto la tasa de gestación en el primer servicio como la tasa de gestación acumulada en el día 120 posparto, fueron menores en las vacas con endometritis subclínica. Este dato es congruente con la mayoría de los estudios; aunque en algunos trabajos no encontraron efecto en la fertilidad ${ }^{(6,8,26)}$, en la mayoría de estudios sí se han observado consecuencias negativas en la tasa de gestación en el primer servicio y un aumento del periodo del parto a la concepción ${ }^{(7,14,17)}$. Se han propuesto diversos mecanismos por los cuales la endometritis subclínica afecta la tasa de gestación. Sheldon et al $l^{(3)}$, mencionan que el proceso inflamatorio del endometrio altera el transporte espermático y del embrión, y el proceso de implantación. Asimismo, las endotoxinas y prostaglandinas liberadas durante el proceso inflamatorio afectarían el desarrollo temprano del embrión ${ }^{(27-29)}$; observaron también que las vacas con endometritis subclínica tienen menores concentraciones de estradiol en los folículos ováricos, lo cual presumen afectaría la competencia del ovocito. Un dato interesante es el incremento de muerte embrionaria o fetal en vacas a las cuales se les diagnosticó endometritis subclínica en el día 46 posparto; en estas vacas además de mostrar menor tasa de gestación en el primer servicio, tuvieron alta incidencia de pérdidas de gestaciones (9.6 vs $43.9 \%$; vacas normales y con endometritis subclínica, respectivamente) entre los días 32 y 60 después de la inseminación ${ }^{(5)}$. Así, cualquiera de estos factores o la combinación de ellos pudo determinar la baja tasa de gestación en el primer servicio observada en el presente estudio. La diferencia observada en la tasa de gestación acumulada en día 120 pudo ser consecuencia de persistencia de la endometritis subclínica, ya que en algunas vacas persiste el proceso inflamatorio ${ }^{(5)}$; además, existen efectos residuales de las patologías uterinas posparto en el aparato reproductor que afectan la probabilidad de gestación en los servicios 
subsiguientes $^{(30)}$.

\section{"Conclusiones e implicaciones \|}

Se concluye que la prevalencia de endometritis subclínica en vacas lecheras de la región central de México es del $43 \%$ entre los días 35 y 45 posparto, lo cual disminuyó $11.4 \%$ la tasa de gestación en el primer servicio y $13.7 \%$ la tasa acumulada de gestaciones en el día 120 posparto. Este es el primer estudio en el cual se determina la prevalencia de endometritis subclínica en México y su efecto en la tasa de gestación. El crecimiento de los hatos lecheros observado en los últimos 25 años ha dificultado el examen clínico reproductivo posparto y la administración oportuna de tratamientos. De acuerdo con los resultados del presente estudio, es probable que una causa importante de la baja fertilidad observada en las vacas lecheras tenga su origen en la salud uterina. Por tanto, es necesario realizar estudios para desarrollar técnicas de diagnóstico de endometritis subclínica más sencillas y evaluar tratamientos para mitigar su impacto en la eficiencia reproductiva.

\section{Agradecimientos}

Este estudio fue financiado en parte por el proyecto IN219811-3 y por el Programa de Apoyos para la Superación del Personal Académico (PASPA-DGAPA) de la Universidad Nacional Autónoma de México. Se agradece a los propietarios de los establos del Complejo Lechero de Tizayuca, Hgo. y a los trabajadores, su apoyo para realizar este estudio.

\section{- Literatura citada}

1. Fourichon C, Seegers H, Malher X. Effect of disease on reproduction in the dairy cow: a meta-analysis. Theriogenology 2000;53:1729-1759. 
2. LeBlanc SJ, Duffield TF, Leslie KE, Bateman KG, Keefe GP, Walton JS, et al. Defining and diagnosing postpartum clinical endometritis and its impact on reproductive performance in dairy cows. J Dairy Sci 2002;85:2223-2236.

3. Sheldon IM, Cronin J, Goetze L, Donofrio G, Schuberth HJ. Defining postpartum uterine disease and the mechanisms of infection and immunity in the female reproductive tract in cattle. Biol Reprod 2009;81:1025-1032.

4. Sheldon IM, Lewis GS, LeBlanc S, Gilbert RO. Defining postpartum uterine disease in cattle. Theriogenology 2006;65:1516-1530.

5. Lima FS, Bisinotto RS, Ribeiro ES, Greco LF, Ayres H, Favoreto MG, et al. Effects of 1 or 2 treatments with prostaglandin $\mathrm{F}_{2} \alpha$ on subclinical endometritis and fertility in lactating dairy cows inseminated by timed artificial insemination. J Dairy Sci 2013;96:6480-6488.

6. Madoz LV, Giuliodori MJ, Migliorisi AL, Jaureguiberry M, de la Sota RL. Endometrial cytology, biopsy, and bacteriology for the diagnosis of subclinical endometritis in grazing dairy cows. J Dairy Sci 2014;97:195-201.

7. Kasimanickam R, Duffield TF, Foster RA, Gartley CJ, Leslie KE, Walton JS, et al. Endometrial cytology and ultrasonography for the detection of subclinical endometritis in postpartum dairy cows. Theriogenology 2004;62:9-23.

8. Plöntzke J, Madoz LV, de la Sota RL, Drillich M, Heuwieser W. Subclinical endometritis and its impact on reproductive performance in grazing dairy cattle in Argentina. Anim Reprod Sci 2010;122:52-57.

9. Cheong SH, Nydam DV, Galvão KN, Crosier BM, Gilbert RO. Cow-level and herdlevel risk factors for subclinical endometritis in lactating Holstein cows. J Dairy Sci 2011;94:762-770.

10. Madoz LV, Giuliodori MJ, Jaureguiberry M, Plöntzke J, Drillich M, de la Sota RL. The relationship between endometrial cytology during estrous cycle and cutoff points for the diagnosis of subclinical endometritis in grazing dairy cows. J Dairy Sci 2013;96:43334339.

11. NRC. National Research Council. Nutrient Requirements of Dairy Cattle. $7^{\text {th }}$ revised edition. Washington, DC, USA: National Academy Press; 2001.

12. Dohoo IR, Martin W, Stryhn HE. Veterinary epidemiologic research. Charlottetown, PEI: University of Prince Edward Island; 2003.

13. Systat. Sigmaplot User's Guide, version 10.0. Systat Software Inc., Chicago, IL. 2006. 
14. SAS.SAS User`s Guide: Statistic (Version 9.3). Cary NC, USA Inst. Inc., 2010.

15. Gilbert RO, Shin ST, Guard CL, Erb HN, Frajblat M. Prevalence of endometritis and its effects on reproductive performance of dairy cows. Theriogenology 2005; 64:18791888 .

16. LeBlanc SJ, Osawa T, Dubuc J. Reproductive tract defense and disease in postpartum dairy cows. Theriogenology 2011;76:1610-1618.

17. Galvão KN, Frajblat M, Brittin SB, Butler WR, Guard CL, Gilbert RO. Effect of prostaglandin F2alpha on subclinical endometritis and fertility in dairy cows. J Dairy Sci 2009;92:4906-4913.

18. Sens A, Heuwieser W. Presence of Escherichia coli, Trueperella pyogenes, $\alpha$-hemolytic streptococci, and coagulase-negative staphylococci and prevalence of subclinical endometritis. J Dairy Sci 2013;96:6347-6354.

19. Kim IH, Kang HG, Jeong JK, Hur TY, Jung YH. Inflammatory cytokine concentrations in uterine flush and serum samples from dairy cows with clinical or subclinical endometritis. Theriogenology 2014;82:427-432.

20. Roche JR, Kay JK, Friggens NC, Loor JJ, Berry DP. Assessing and managing body condition score for the prevention of metabolic disease in dairy cows. Vet Clin North Am Food Anim Pract 2013;29:323-336.

21. Giuliodori MJ, Magnasco RP, Becu-Villalobos D, Lacau-Mengido IM, Risco CA, de la Sota RL. Metritis in dairy cows: risk factors and reproductive performance. J Dairy Sci 2013;96:3621-3631.

22. Santos NR, Lamb GC, Brown DR, Gilbert RO. Postpartum endometrial cytology in beef cows. Theriogenology 2009;71:739-745.

23. Ricci A, Gallo S, Molinaro F, Dondo A, Zoppi S, Vincenti L. Evaluation of subclinical endometritis and consequences on fertility in Piedmontese beef cows. Reprod Domest Anim 2015;50:142-148.

24. Bacha B, Regassa FG. Subclinical endometritis in Zebu x Friesian crossbred dairy cows: its risk factors, association with subclinical mastitis and effect on reproductive performance. Trop Anim Health Prod 2010;42:397-403.

25. Carneiro LC, Ferreira AF, Padua M, Saut JP, Ferraudo AS, dos Santos RM. Incidence of subclinical endometritis and its effects on reproductive performance of crossbred dairy cows. Trop Anim Health Prod 2014;46:1435-1439.

26. Kasimanickam R, Cornwell JM, Nebel RL. Effect of presence of clinical and subclinical 
endometritis at the initiation of Presynch-Ovsynch program on the first service pregnancy in dairy cows. Anim Reprod Sci 2006;95:214-223.

27. Hill J, Gilbert R. Reduced quality of bovine embryos cultured in media conditioned by exposure to an inflamed endometrium. Aust Vet J 2008;86:312-316.

28. Green MP, Ledgard AM, Beaumont SE, Berg MC, McNatty KP, Peterson AJ, et al. Long-term alteration of follicular steroid concentrations in relation to subclinical endometritis in postpartum dairy cows. J Anim Sci 2011;89:3551-3560.

29. Carneiro LC, Cronin JG, Sheldon IM. Mechanisms linking bacterial infections of the bovine endometrium to disease and infertility. Reprod Biol 2016;16:1-7.

30. Ribeiro ES, Gomes G, Greco LF, Cerri RL, Vieira-Neto A, Monteiro PL Jr, et al. Carryover effect of postpartum inflammatory diseases on developmental biology and fertility in lactating dairy cows. J Dairy Sci 2016;99:2201-2220. 